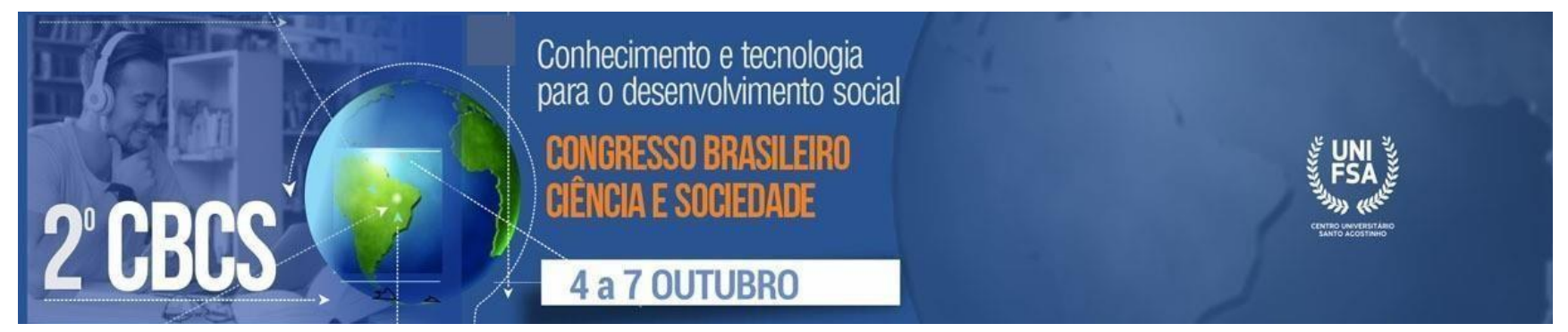

\title{
IMPACTO DA PANDEMIA DE COVID-19 NAS FAMÍLIAS DE PACIENTES EM CUIDADOS INTENSIVOS E ESTRATÉGIAS DE ENFRENTAMENTO: UMA REVISÃO DE ESCOPO ${ }^{1}$
}

\author{
Giovana Moura Nogueira², UNIFSA \\ Vanessa Maria Alves de Andrade ${ }^{3}$, UNIFSA \\ Willyane de Andrade Alvarenga ${ }^{4}$, UNIFSA \\ Lucila Castanheiro Nascimento ${ }^{5}$, EERP-USP \\ Silvana Santiago da Rocha ${ }^{6}$, UFPI
}

\section{RESUMO}

Diante das mudanças estruturais e nos cuidados decorrentes da pandemia de COVID-19, há a necessidade de conhecer os impactos causados às famílias de pacientes internados em unidades de terapia intensiva (UTI). Objetivo: mapear evidências científicas sobre o impacto familiar relacionado à internação de um ente querido por COVID-19 em UTI e as estratégias de enfrentamento dos serviços para minimizar esses impactos. Método: revisão de escopo, realizada nas bases de dados PubMed e LILACS. Resultados: Foram encontrados 410 estudos e incluídos 12 estudos na amostra final. Impactos emocionais, psicológico e sociais foram relados e tecnologias de comunicação com a família puderam reduzir esses impactos, tais como o uso de vídeo conferência, diário de UTI, programas, mensagens, notas e chamadas telefônicas. Conclusão: A família é emocionalmente desafiada pela internação do familiar na UTI e pelas restrições decorrentes da COVID-19. Estratégias podem ser utilizadas pela equipe de saúde para reduzir os impactos causados.

Palavras-Chave: Sars Cov-19. Família. UTI. Revisão

\footnotetext{
${ }^{1}$ Trabalho apresentado no 2ㅇ Congresso Brasileiro Ciência e Sociedade (CBCS 2021), promovido pelo Centro Universitário Santo Agostinho, de 04 a 07 de outubro de 2021, em Teresina-PI.

${ }^{2}$ Estudante de graduação em Enfermagem do Centro Universitário Santo Agostinho (UNIFSA). E-mail: giovananogueiram@gmail.com

${ }^{3}$ Estudante de graduação em Enfermagem do Centro Universitário Santo Agostinho (UNIFSA). E-mail: contatov.andrademakeup@gmail.com

${ }^{4}$ Doutora em Enfermagem. Professora do Centro Universitário Santo Agostinho (UNIFSA). E-mail: willyalvarenga@unifsa.com.br

${ }^{5}$ Doutora em Enfermagem. Professora Titular da Escola de Enfermagem de Ribeirão Preto da Universidade de São Paulo (EERP-USP). E-mail: lucila@eerp.usp.br

${ }^{6}$ Doutora em Enfermagem. Professora Adjunta da Universidade Federal do Piauí (UFPI). E-mail: silvanasantiago27@gmail.com
} 


\section{INTRODUÇÃO}

A infecção causada SARS-CoV-2/2019-nCnV surgiu em Wuhan, província de Hubei, China, ao final de 2019, como uma síndrome respiratória aguda grave, que ficou conhecida como corona vírus (COVID-19) (CAl et al., 2020). Em 26 de fevereiro de 2020, foi confirmado o primeiro caso do vírus no Brasil e ao início de março, a primeira morte; ambas em São Paulo (BRASIL, 2020).

Já são confirmados mais 119.515.052 casos de COVID-19 no mundo, o Brasil se encontra entre os países com maior número de casos, com 11.439.558 casos, atrás apenas dos Estados Unidos. Já em relação aos óbitos, o Brasil registrou o maior número de óbitos novos em todo mundo, alcançando 12.777 de perdas (BRASIL, 2021). Isso é justificado pelo grande índice de disseminação, que ocorre principalmente pelo contato direto ou por gotículas espalhadas pela tosse, ou espirro de um indivíduo infectado (ROTHAN; BYRAREDDY, 2020). Além disso, há as pessoas assintomáticas, que também transmitem o vírus (OPAS, 2021).

A situação da pandemia de COVID-19 revelou uma série de preocupações aos sistemas e os prestadores de cuidados de saúde, no que se refere ao desperdício de recursos, adaptação dos ambientes, perda inadvertida de vidas e perda da confiança de usuários e profissionais de saúde (EMANUEL et al., 2020). Além disso, a situação fez retroceder anos das políticas de visitação nas unidades de terapia intensiva (UTI) que estavam ampliando a participação das famílias no cuidado aos pacientes, devido às medidas restritivas e de bloqueio ao acesso às UTIs para proteção dos pacientes e de seus familiares (NUNES, 2020). Assim as informações como o boletim diário e o contato, que antes era feito pessoalmente com o familiar, foram alteradas para ligações telefônicas e vídeo chamadas (FIRMINO et al., 2015).

Além disso, outras mudanças foram necessárias como o aumento na quantidade de leitos de UTI destinados às pessoas contaminadas pelo novo corona vírus, que aumentou de 46.045 mil no período pré-pandemia para 60.265 leitos no Brasil, significando um aumento expressivo para o cenário nacional (COTRIM; CABRAL, 2020). Enfermeiros e demais profissionais de saúde tiveram que passar por treinamentos de novos protocolos e, paralelamente, havia grande procura por leitos de UTI, que se tornavam insuficiente para todos os pacientes, deixando familiares ainda mais aflitos (SOUZA; DE SOUSA, 2021). 
Diferentes impactos à família são relatados na literatura com a internação de um membro familiar em uma UTI. Um misto de sentimentos como esperança, alívio, insegurança e temor podem surgir, pois a doença e a hospitalização representam a ruptura do cotidiano, perda dos vínculos com o trabalho, a família, os amigos e as demais áreas da vida, o que pode gerar ansiedade e estresse (OTHERO; CARLO; 2006). A internação na UTI é ainda mais estressante, pois é um local destinados ao cuidado de pacientes graves que, quase sempre, é associado à morte e ao sofrimento (PROENÇA; AGNOLLO, 2011). A confiança da família na equipe de saúde é imprescindível e só é conseguida com uma boa relação comunicativa e o respeito aos valores da família (RESTELATTO; HOFFELDER, 2018).

Nenhuma revisão de literatura foi encontrada sobre o impacto na família relacionado à internação de um ente querido em unidade de cuidados intensivos em tempos de pandemia de COVID-19 e que estratégias as UTIs realizaram para lidar com as mudança que foram exigidas diante desse cenário. Estes resultados poderão dar visibilidade às experiências das famílias e às estratégias utilizadas para mitigar os impactos decorrentes das mudanças estruturais e de cuidado críticos decorrentes da pandemia. Assim, essa breve revisão de escopo objetivou mapear evidências científicas sobre o impacto familiar relacionado à internação de um ente querido em unidade de cuidados intensivos por COVID-19 e as estratégias de enfrentamento dos serviços para minimizar esses impactos. Esse estudo buscou responder as seguintes questões de pesquisa: Como a internação de um ente querido em unidade de cuidados intensivos em tempos de COVID-19 impacta as famílias? e Quais as estratégias utilizadas pela equipe de cuidados intensivos para minimizar esses impactos?

\section{METODOLOGIA}

Esta é uma breve revisão de escopo (Scoping Review). Este tipo de revisão objetiva mapear rapidamente os conceitos-chave que sustentam uma área de pesquisa, as principais fontes e tipos de evidências disponíveis, especialmente em uma área complexa ou que não foi revisado antes (MAYS; ROBERS; POPAY, 2001). Foram percorridas cinco fases que concernem: 1. Identificar a questão da pesquisa; 2. Identificação de estudos relevantes; 3. Seleção do estudo; 4. Mapeamento dos dados; 5. Agrupar, resumir, e relatar os estudos (ARKSY; O'MALLEY, 2003). O Reporting Items 
for Systematic Reviews and Meta-Analyzes extensions for Scoping Reviews (PRISMA-ScR) foi usado para guiar e reportar os itens essenciais desta revisão (TRICCO et al., 2018).

Foram incluídos estudos primários de abordagem qualitativa, quantitativa, misto/multimétodos ou relatos de experiência; com profissionais de saúde ou membros familiares que enfocaram o impacto na família tanto da notícia da necessidade de internação do membro familiar em uma unidade de cuidados intensivos como da vivência/experiência dessa internação em tempos de COVID-19. Artigos científicos publicados em português, inglês e espanhol, nos últimos dois anos (período quando a infecção pelo novo cora vírus foi documentada) e independente da região geográfica foram incluídos. Foram excluídos editoriais, dissertações, teses, resumos de anais de congressos, livro e capítulo de livro, textos incompletos, ou estudos sobre a vivência do paciente ou da família em outros contextos de internação.

Foram realizadas buscas nas base de PubMed (American psychologiacal association) e LILACS (Literatura Latino-americana e do Caribe em CiênciaNational Library of Medicine). A ferramenta PCC (P: População, C: Conceito e C: Contexto) foi utilizada para elaborar a questão de pesquisa e a estratégia de busca (ARKEY; O'MALLEY,2005). Descritores (Mesh e DeCs) e palavra-chaves foram combinados pelos booleanos AND e OR. Exemplo da estratégia do PubMed está contido na tabela 1 e foi adaptada para a base de dados LILACS. Artigos identificados por meio da busca manual nas referências dos artigos da amostra final foram incluídos.

Quadro 1. Estratégia de busca com os termos utilizados na PubMed.

\begin{tabular}{|c|c|c|}
\hline PCC & DESCRIÇÃO & TERMOS DE BUSCA \\
\hline P: População & Família & $\begin{array}{l}\text { "Family"* Mesh+ OR (Family) OR "Mothers"[Mesh] OR } \\
\text { (Mother) OR "Fathers"[Mesh] OR (Father) OR } \\
\text { "Grandparents"[Mesh] OR (Grandparent) OR } \\
\text { (Grandmother) OR (Grandmothers) OR (Grandfather) OR } \\
\text { (Grandfathers) OR "Siblings"[Mesh] OR (Sibling) OR (Sisters) } \\
\text { OR (Sister) OR (Brothers) OR (Brother) OR (Family Research) } \\
\text { OR (Research, Family) OR (Relatives) OR (Extended Family) } \\
\text { OR (Extended Families) OR (Families, Extended) OR (Family, } \\
\text { Extended) OR (Family Research) OR (Family Members) OR } \\
\text { (Family Member) }\end{array}$ \\
\hline C: Conceito & $\begin{array}{l}\text { Experiência de } \\
\text { internação em } \\
\text { unidades de } \\
\text { cuidado intensiva }\end{array}$ & $\begin{array}{l}\text { "Intensive Care Units"[Mesh] OR (ICU) OR } \\
\text { (Intensive Care Unit) OR (Unit, Intensive Care) OR (ICU } \\
\text { Intensive Care Unit) OR lived experience* or life experience } \\
\text { or experiences or feeling* OR mental OR emotion* OR } \\
\text { psychol* }\end{array}$ \\
\hline C: Contexto & $\begin{array}{l}\text { Infecções por } \\
\text { Coronavírus }\end{array}$ & $\begin{array}{l}\text { "COVID-19"*Mesh+ OR (Coronavírus Infections) OR (COVID- } \\
\text { 19) OR (COVID-19 Virus Disease) OR (COVID } 19 \text { Virus } \\
\text { Disease) OR (COVID-19 Virus Diseases) OR (COVID- } \\
19 \text { Pandemic) OR (COVID } 19 \text { Pandemic) OR (COVID- } \\
19 \text { Pandemics) OR (Pandemic, COVID-19) OR (SARS } \\
\text { Coronavirus } 2 \text { Infection) OR (SARS-CoV-2 Infection) OR } \\
\text { (Infection, SARS-CoV-2) OR (SARS CoV } 2 \text { Infection) OR (SARS- } \\
\text { CoV-2 Infections) OR (2019 Novel Coronavirus Disease) OR } \\
\text { (2019 Novel Coronavirus Infection) OR (2019-nCoV Disease) } \\
\text { OR (2019 nCoV Disease) OR (2019- } \\
\text { nCoV Diseases) OR (Disease, 2019-nCoV) }\end{array}$ \\
\hline
\end{tabular}

Fonte: Elaborado pelas autoras 
O processo de seleção dos estudos foi realizado por dois revisores de forma independente. Foi utilizado o website Ryan QCRI, para onde foram enviados os estudos que foram avaliados individualmente por dois revisores. Inicialmente foram excluídos os estudos duplicados. Feito isso, a partir da leitura dos títulos e resumos dos estudos foram removidos todos que não atendiam aos critérios de elegibilidade. Os conflitos que haviam entre os dois revisores foram resolvidos por uma terceira revisora. Os estudos selecionados na primeira etapa foram lidos na íntegra individualmente por todos os revisores, de forma independente. O fluxograma do PRISMA (Figura 1) foi utilizado para reportar o processo de seleção dos estudos (PAGE et al., 2021)

Informações sobre ano das publicações, objetivo, método, principais resultados e características das famílias e pacientes foram extraídos dos estudos incluídos, considerando um formulário elaborado pelas autoras. Os resultados dos estudos incluídos foram analisados pelos dois revisores para extrair informações sobre o impacto familiar e as estratégias de enfretamento diante da internação de um ente querido em unidade de cuidados intensivos por COVID-19. Uma tabela de caracterização dos estudos e temas descritivos foram construídos e validados por todos os autores para apresentar os resultados desta revisão.

\section{RESULTADOS E DISCUSSÃO}

Foram encontrados 410 estudos, 402 a partir das bases de dados e 8 a partir da busca manual, após a analise dos estudos incluídos. Excluíram-se 12 artigos por estarem duplicados, restando-se 390 artigos, que tiveram seus títulos e resumos analisados com base nos critérios de inclusão, por dois revisores de forma independente. Foram incluídos 33 artigos após a triagem e 21 artigos foram lidos na íntegra por dois revisores, independentemente, resultando em 10 artigos excluídos, pois não focavam na família e no paciente internado na UTI por conta do COVID 19, estavam incompletos ou não foram recuperados Assim a amostra final desta revisão dos de 12 artigos (Figura 1). 
FIGURA 1. Fluxograma PRISMA do processo de busca na literatura.

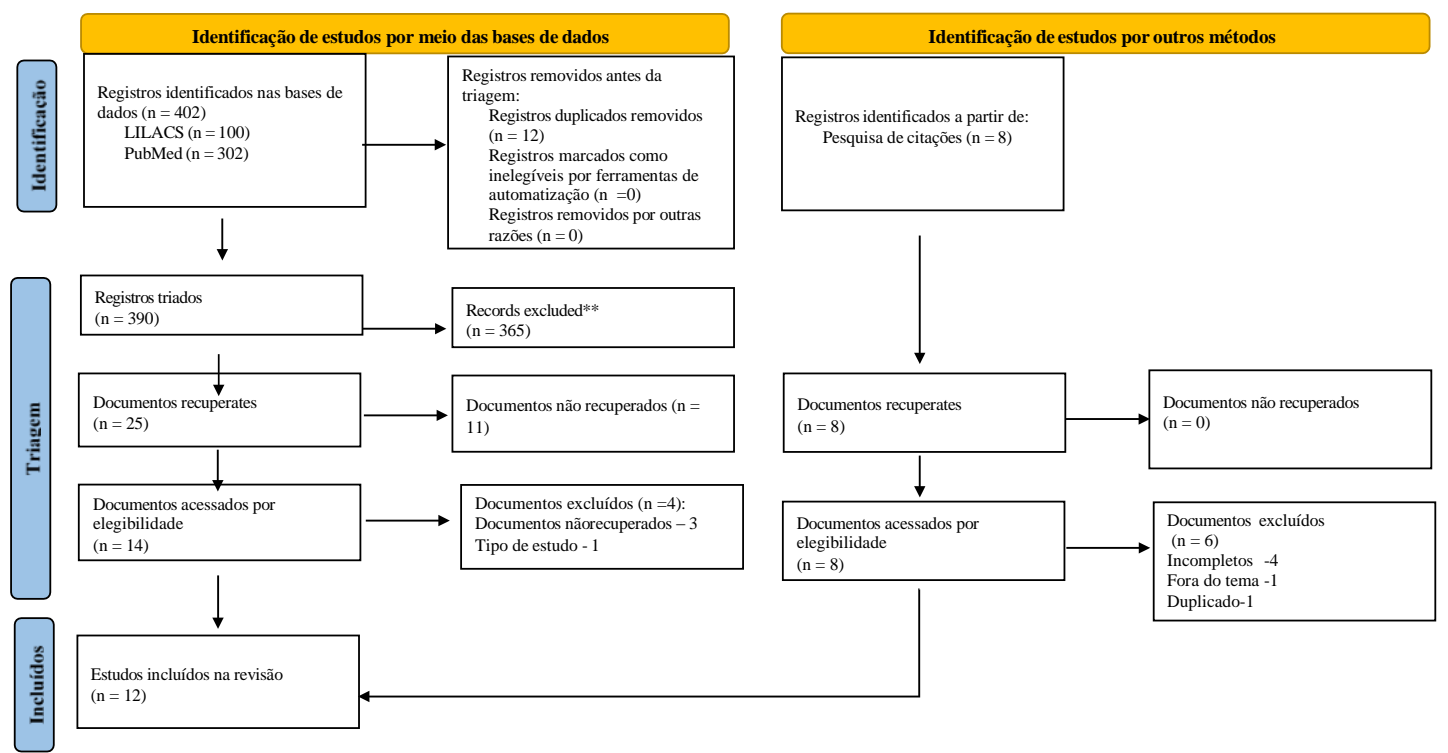

Fonte: Adaptado de PAGE et al. (2021).

\section{Características dos estudos incluídos}

As caraterísticas dos estudos incluídos estão apresentadas no Quadro 2. Os estudos que compõem a amostra $(n=12)$ foram desenvolvidos, em sua maioria, nos Estados Unidos ( $n=5,39,9 \%$ ) e um na China, Japão, Irã, Holanda, Itália, Reino Unido e Países Baixos. Desses artigos, seis eram estudos quantitativos do tipo coorte $(n=3)$, observacional retrospectivo $(n=1)$, transversal $(n=1)$ e ensaio clinico randomizado $(n=1)$, dois eram estudos qualitativos, do tipo exploratório $(n=1)$ e fenomenológico $(n=1)$, três estudo de caso e um relato de experiência. Os estudos incluídos apresentam dados de 2374 participantes. O número mínimo de participantes nos estudos foi seis e o numero máximo de participantes foi 2000. A maioria de pacientes era do sexo masculino e seus familiares do sexo feminino, principalmente companheira/esposas e filhas. 
QUADRO 2. Características dos estudos inclusos, de acordo com primeiro autor, ano e pais, objetivos, método e participantes.

\begin{tabular}{|c|c|c|c|}
\hline $\begin{array}{c}\text { AUTOR (ano) } \\
\text { país }\end{array}$ & OBJETIVO & MÉTODO & $\begin{array}{l}\text { CARACTERISTICAS DOS } \\
\text { PACIENTES E FAMILIARES }\end{array}$ \\
\hline $\begin{array}{l}\text { PISCITELLO et } \\
\text { al,(2021) Estados } \\
\text { Unidos }\end{array}$ & $\begin{array}{l}\text { Avaliar a quantidade de reuniões } \\
\text { familiares por telefone, vídeo e } \\
\text { presencial durante a pandemia de } \\
\text { COVID-19 por gráfico manual. }\end{array}$ & Estudo de coorte & $\begin{array}{l}\text { Pacientes COVID-19 admitidos na } \\
\text { UTI foram total de } 57 \text {, a maioria } \\
\text { dos pacientes eram hispânicos ou } \\
\text { latinos, o período de estudo foi } \\
\text { de três meses entre } 27 \text { de março e } \\
30 \text { de junho de } 2020 \text {, } \\
\end{array}$ \\
\hline $\begin{array}{l}\text { LIU et al,(2020) } \\
\text { China }\end{array}$ & $\begin{array}{l}\text { Descrever a importância de } \\
\text { acompanhantes de membros da } \\
\text { família com COVID-19 e seu } \\
\text { benefício potencial para o } \\
\text { tratamento da doença. }\end{array}$ & $\begin{array}{l}\text { Estudo } \\
\text { observacional } \\
\text { retrospectivo }\end{array}$ & $\begin{array}{l}\text { Pacientes admitidos entre } 9 \text { e } 29 \\
\text { de fevereiro de } 2020 \text {, com covidD- } \\
19,53 \text { foram incluídos na análise } \\
\text { final. }\end{array}$ \\
\hline $\begin{array}{l}\text { LIPWORTH et } \\
\text { al,(2021) Estados } \\
\text { Unidos }\end{array}$ & $\begin{array}{l}\text { Relatar, os elementos primários } \\
\text { necessários para reproduzir e } \\
\text { dimensionar o Programa de Apoio } \\
\text { aos Pacientes COVID-19 em } \\
\text { hospitais que enfrentam surtos } \\
\text { semelhantes de UTI, incluindo uma } \\
\text { lista de verificação para replicação }\end{array}$ & $\begin{array}{l}\text { Relato de } \\
\text { experiência }\end{array}$ & $\begin{array}{l}\text { Havia } 65 \text { funcionários, no total de } \\
19 \text { especialidades diferentes que } \\
\text { mantinham 140 familiares de } \\
\text { pacientes da UTI positivos para } \\
\text { COVID } 19 \text { informados sobre o } \\
\text { estado de saúde dos seus entes }\end{array}$ \\
\hline $\begin{array}{l}\text { VEENENDAAL et } \\
\text { al, (2021) Países } \\
\text { Baixos }\end{array}$ & $\begin{array}{l}\text { Obter uma visão sobre a longo } \\
\text { prazo funcionamento físico, social e } \\
\text { psicológico de sobreviventes da UTI } \\
\text { COVID-19 e seus familiares trêse } \\
\text { seis meses após a alta da UTI. }\end{array}$ & $\begin{array}{l}\text { Coorte prospectiva } \\
\text { de centro único }\end{array}$ & $\begin{array}{l}\text { Foram } 94 \text { pacientes internados na } \\
\text { UTI, no qual foram convidados } \\
102 \text { membros da família para } \\
\text { participar desse estudo e apenas } \\
67(66 \%) \text { no total aceitaram. O } \\
\text { tempomedio de permanência na } \\
\text { UTI foram de } 19,4 \text { dias, a maioria } \\
\text { dos familiares eram companheiras } \\
\text { dos pacientes, sendo do sexo } \\
\text { feminino onde a idade media era } \\
56 \text { anos. }\end{array}$ \\
\hline $\begin{array}{l}\text { CHEN et al, } \\
\text { (2021) Estados } \\
\text { Unidos }\end{array}$ & $\begin{array}{l}\text { Explorar as experiências e } \\
\text { necessidades de apoio dos } \\
\text { familiares de pacientes com coviD- } \\
19 \text { ventilados na unidade de terapia } \\
\text { intensiva (UTI). }\end{array}$ & $\begin{array}{l}\text { Exploratório, } \\
\text { qualitativo }\end{array}$ & $\begin{array}{l}\text { O numero foi de } 10 \text { membros de } \\
\text { famílias de pacientes adultos com } \\
\text { COVID-19 na UTI que tinham em } \\
\text { media 51 anos, relação do } \\
\text { membro da família com o } \\
\text { paciente } 3 \text { cônjuges, } 3 \text { filhos, } 2 \\
\text { pais, } 1 \text { sobrinho e } 1 \text { sobrinha). }\end{array}$ \\
\hline $\begin{array}{l}\text { WENDEL et al, } \\
\text { (2020) Estados } \\
\text { Unidos. }\end{array}$ & $\begin{array}{l}\text { Fornecer suporte abrangente às } \\
\text { famílias de pacientes COVID-19, e } \\
\text { para criar capacidade para que } \\
\text { nossas equipes de UTI se } \\
\text { concentrem no atendimento ao } \\
\text { paciente. Neste breve relatório, } \\
\text { descrevemoso o desenvolvimento, } \\
\text { implementação e experiência } \\
\text { preliminar com o serviço. }\end{array}$ & Estudo de caso & \begin{tabular}{lrcc|} 
Incluíram & 20 & familiares & de \\
pacientes que & não tinham & ou \\
tinham poucas informações & por \\
dia dos seus entes. & & \\
la &
\end{tabular} \\
\hline $\begin{array}{l}\text { ROSE et al,(2021) } \\
\text { Reino Unido }\end{array}$ & $\begin{array}{l}\text { Entender como a comunicação } \\
\text { entre famílias, paciente e a equipe } \\
\text { da UTI foram capacitadas durante a } \\
\text { pandemia. Os objetivos } \\
\text { secundários eram compreender } \\
\text { estratégias usadas para facilitar a } \\
\text { visita virtual e benefícios e } \\
\text { barreiras associados. }\end{array}$ & $\begin{array}{l}\text { Pesquisa eletrônica } \\
\text { multicêntrica, } \\
\text { transversal e auto- } \\
\text { administrada. }\end{array}$ & $\begin{array}{l}182 \text { UTIs impuseram restriçōes de } \\
\text { visitas, destas } 28(16 \%) \text { não } \\
\text { permitiam em nenhuma } \\
\text { circunstância visitas, já } 112(63 \% \\
\text { das UUtis permitiam apresena da } \\
\text { família no fina da vida, } 106(50 \%) \\
\text { estabeleceram uma equipe } \\
\text { dedicada aligações para a família }\end{array}$ \\
\hline $\begin{array}{l}\text { SHARIATI et } \\
a l,(2021) \text { Irã }\end{array}$ & $\begin{array}{l}\text { Identificar o efeito da comunicação } \\
\text { baseada na web entre uma } \\
\text { enfermeira e um membro da } \\
\text { família de um paciente com } \\
\text { COVID19 em seu estresse } \\
\text { percebido. }\end{array}$ & Ensaio clinico & $\begin{array}{l}67 \text { familiares de pacientes com } \\
\text { COVID19 internados na UTI de } \\
\text { maio a agosto de } 2020 \text { referentes } \\
\text { a três dos hospitais no Irã }\end{array}$ \\
\hline $\begin{array}{l}\text { BARTOLI et al, } \\
\text { (2021) Itália }\end{array}$ & $\begin{array}{l}\text { Descrever as experiências vividas } \\
\text { por famílias com membro } \\
\text { internado em COVID-UTI }\end{array}$ & $\begin{array}{l}\text { Estudo } \\
\text { fenomenológico }\end{array}$ & $\begin{array}{l}14 \text { familiares de primeiro grau } \\
\text { foram contatados por telefones } \\
24 \text { hrs após a admissão de seus } \\
\text { entes, destes } 2 \text { (14\%) eram } \\
\text { homens e 12(86\%) mulheres } \\
\text { (7fihas, } 4 \text { irmãs, } 1 \text { esposa) com a } \\
\text { idade media de } 47 \text { á } 57 \text {. }\end{array}$ \\
\hline $\begin{array}{l}\text { LEMMERS; } \\
\text { VOORT (2021) } \\
\text { Holanda }\end{array}$ & $\begin{array}{l}\text { Desenvolver uma estrutura } \\
\text { conceitual de confiança entre HCPs } \\
\text { e pacientes com suas famílias que } \\
\text { pode ser aplicado ao ambiente de } \\
\text { terapia intensiva. }\end{array}$ & Estudo de caso & $\begin{array}{l}\text { A UTI em OLVG- leste onde } 2000 \\
\text { pacientes são tratados por ano a } \\
\text { media de idade dos pacientes é de } \\
68 \text { anos,1200 }(60 \%) \text { são do sexo } \\
\text { masculino e } 800(40 \%) \text { são do } \\
\text { sexo feminino. }\end{array}$ \\
\hline $\begin{array}{l}\text { PISCITELLO et al, } \\
\text { (2020) Estados } \\
\text { Unidos }\end{array}$ & $\begin{array}{l}\text { Mostrar e avaliar a quantidade de } \\
\text { reuniōes familiares por telefone, } \\
\text { vídeo e presencial durante a } \\
\text { pandemia de COVID-19 por gráfico } \\
\text { manual. }\end{array}$ & $\begin{array}{l}\text { Estudo de coorte } \\
\text { retrospectivo }\end{array}$ & $\begin{array}{l}61 \text { pacientes incluídos neste } \\
\text { estudo a maioria dos pacientes } \\
\text { eram hispânicos ou latinos ( } \mathrm{n} \% / 4 \\
32 / 61,62 \% \text { ) com o tempo médio } \\
\text { de internação de } 23,5 \text { dias (DP } \\
14,0) \text {. }\end{array}$ \\
\hline $\begin{array}{l}\text { HARUNA et al, } \\
\text { (2021) Japão. }\end{array}$ & $\begin{array}{l}\text { Analisar a utilidade de um diário de } \\
\text { unidade de terapia intensiva (UTI) } \\
\text { sobre as vivências de familiares de } \\
\text { CovID-19 crítico. }\end{array}$ & Estudo de caso & $\begin{array}{l}\text { Incluídos } 2 \text { pacientes de UTI } \\
\text { CovID do sexo masculino, } 4 \\
\text { familiares todas mulheres, }\end{array}$ \\
\hline
\end{tabular}

Fonte: elaborado pelas autoras 
Os artigos a cima evidenciam que pandemia trouxe diversas consequencias há qualidade física, social e psicológica de familiares de pacientes internados na Unidade de Terapia Intensiva, o que fez serem utilizadas varias medidas para que fosse minimizado o sofrimento do seu ente como é demonstrado na FIGURA 2.

FIGURA 2. Impacto familiar das restrições relacionadas à pandemia COVID-19 e tecnologias de comunicação para minimizar os impactos.

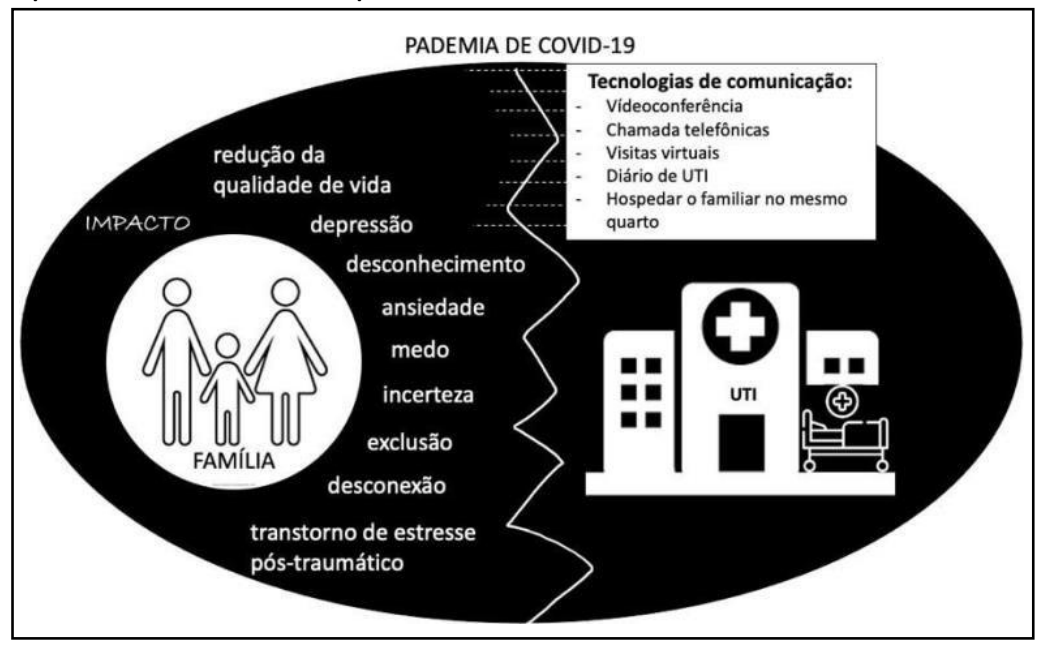

Fonte: Elaborado pelas autoras

\section{Impacto físico, social e psicológico de familiares}

O impacto da admissão na UTI vai além do paciente. As restrições aos visitantes causaram desafios para os familiares devido a condição física instável de seus pacientes, situações de risco de vida, como insuficiência respiratória, medo de piorar as condições, ausência da presença, desconhecimento da condição dos pacientes durante a hospitalização, ansiedade quanto à infecção de si próprios e outros familiares, bem como a falta de conhecimento sobre o cuidado ao paciente (SHARIAT et al 2021). Estudos relataram experiências de estresse e a incerteza dos familiares pela incapacidade de se sentirem conectados ao paciente e informados sobre os cuidados, além da necessidade de apoio (CHEN et al 2021; LEMMERS et al 2021). Os familiares se viram preocupados com o quadro clínico dos entes queridos e se sentindo excluídos do processo de cuidar, o que aumentou o medo do futuro (BARTOLI et al 2021).

Sintomas como ansiedade, depressão, transtorno de estresse pós-traumático e redução da qualidade de vida também estiveram presentes em familiares de sobreviventes de UTI (SHARIAT et al. 2021; VEENEDAAL et al 2021). Este fenômeno é conhecido como síndrome pós-terapia intensiva familiar (PICS-F) (VEENEDAAL et al 2021). O impacto psicológico da separação relacionada ao COVID nas famílias de 
pacientes internados na UTI reverberará por anos e resultará em um grande número de pessoas com necessidade de serviços de saúde mental (pessoalmente ou telessaúde ) para aliviar os sintomas e prevenir resultados adversos associados a doenças psiquiátricas não tratadas (por exemplo, uso indevido de substâncias, ideação suicida) (MONTAUK et al 2020).

\section{Estratégias para apoiar familiares e minimizar os impactos}

A comunicação eficaz entre familiares de pacientes criticamente enfermos e equipe é um componente central no cuidado centrado no paciente na UTI (WENDEL et al 2020). Intervenções educativas e de cuidado à distância são uma medida prática e eficaz que foi utilizada de várias formas no cenário de COVID-19 para as restrições às visitas familiares à UTI, incluindo comunicação via web, videoconferência e telefone (PISCITELLO et al 2021; SHARIAT et al 2021). Visitas virtuais e equipes de comunicação dedicadas foram inovações comuns e resultaram em benefícios valiosos em termos de recuperação do paciente e moral da equipe (ROSE et al 2021). A videoconferência foi utilizada para configurar sessões de treinamento e de perguntas e respostas que foram associadas à melhoria da satisfação das famílias (SHARIAT et al 2021).

Programa de comunicação para apoiar a comunicação entre as equipes da UTI, a equipe de cuidados paliativos e as famílias dos pacientes da UTI foram desenvolvidos, em que os contatos de comunicação com as famílias ocorria pelo menos uma vez por dia para fornecer atualizações clínicas oportunas e precisas, orientação empática para decisões difíceis e suporte emocional consistente (LIPWORTH et al 2020). O contato por telefone e vídeo foram avaliados positivamente, pois para muitos familiares (68\%) a distância física era 'muito difícil' de lidar (VEENEDAAL et al 2021). Para muitas famílias, ferramentas de vídeo chamada serviram como a única fonte de conexão com seu ente querido e essa tecnologia deve ser incorporada o mais cedo possível no atendimento (MONTAUK et al 2020). Melhorar o acesso e desenvolver uma abordagem mais consistente para a visita virtual da família à UTI pode melhorar a qualidade do atendimento, tanto durante quanto fora de condições pandêmicas (ROSE et al 2021). Recomendações como compreender e respeitar que algumas famílias podem sentir desconforto ao ver seu ente querido em estado crítico e trabalhar com elas da maneira 
que lhes for mais confortável (por exemplo, recebendo apenas atualizações verbais por telefone) (MONTAUK et al 2020).

Hospedar membros da família no mesmo quarto fornece a alguns pacientes apoio familiar durante o isolamento. Isso pode ser benéfico, pois a companhia e o apoio emocional face a face de membros da família ou colegas sociais podem facilitar a recuperação física (CHEN et al 2021). O diário da UTI pode ser uma ferramenta útil para divulgar informações dos pacientes quando a visita à UTI é restrita devido à pandemia de COVID-19. Comparando as experiências de familiares de pacientes graves com COVID-19, antes e depois do recebimento do diário na UTI, os familiares falavam com ansiedade e pessimismo usando palavras emocionais antes de receber o diário da UTI, entretanto, após o recebimento do diário da UTI, tais experiências emocionais negativas foram atenuadas, resultando em aumento de palavras e sentimentos positivos (HARUNA J. et al 2021).

\section{CONSIDERAÇÕES FINAIS/CONCLUSÕES}

Esta revisão mostrou que o impacto de uma internação na UTI por COVID-19, não acomete apenas o paciente, mais a família como um todo, trazendo impactos nos âmbitos: emocionais, psicológico e sociais. Esse impacto é causado pelo medo, ansiedade e pela incerteza de cura do ente querido. $\mathrm{O}$ isolamento, as restrições de visitas e a falta de informações do ente internado aumentaram ainda mais o medo e falta de confiança acarretando possíveis problema psicológicos, como trauma e estresse pós-traumático tanto aos pacientes quanto aos seus familiares.

Algumas estratégias podem reduzir esses impactos como o uso de vídeo chamada, ou vídeo conferência, diário de UTI, e programas de comunicação com a família por meio de mensagens, notas, chamadas telefônicas, que permite a família ter mais segurança e confiança sobre o estado do paciente e reduzir os impactos causados pela internação do ente na UTI por COVID-19. 


\section{REFERÊNCIAS}

ARKSEY, Hilary; O'Malley, Lisa (2005). Scoping studies: towards a methodological framework. International Journal of Social Research Methodology, 8(1), 19-

32. doi:10.1080/1364557032000119616

BARTOLI D, Trotta F, Simeone S, Pucciarelli G, Orsi GB, Acampora O, Muzio MD, Cappitella C, Rocco M. The lived experiences of family members of Covid-19 patients admitted to intensive care unit: A phenomenological study. Heart Lung. 2021 Aug 7;50(6):926-932. doi:

10.1016/j.hrtlng.2021.08.002. Epub ahead of print. PMID: 34428738; PMCID: PMC8346335.

BRASIL, Ministério da Saúde. Boletim Epidemiológico Especial. Doença pelo Covid-19. Semana Epidemiológica 10 (7/3 a 13/3/2021). Disponível em: https://www.gov.br/saude/pt-

br/media/pdf/2021/marco/18/boletim_epidemiologico_covid_54-1.pdf. Acessado 12/05/2021.

BRASIL, Ministério da Saúde. COVID-19. Painel coronavírus: Ministério da Saúde [ Internet], 2020. Disponível em: https://covid.saude.gov.br/. Acessado 12/05/2021.

CAI, JIANGHUI et al. Allocation of pharmaceutical resources in maternal and child healthcare institutions during the COVID-19 pandemic. Revista da Associação Médica Brasileira, v. 66, p. 41-47, 2020.

CHEN C, Wittenberg E, Sullivan SS, Lorenz RA, Chang YP. The Experiences of Family Members of Ventilated COVID-19 Patients in the Intensive Care Unit: A Qualitative Study. Am J Hosp Palliat Care. 2021 Jul;38(7):869-876. doi: 10.1177/10499091211006914. Epub 2021 Apr 1. PMID: 33789492; PMCID: PMC8020036.

COTRIM JUNIOR, Dorival Fagundes; CABRAL, Lucas Manoel da Silva. Crescimento dos leitos de UTI no país durante a pandemia de Covid-19: desigualdades entre o público x privado e iniquidades regionais. Physis: Revista de Saúde Coletiva, v. 30, p. e300317, 2020.

CRESWELL, John W.; CRESWELL, J. David. Projeto de pesquisa-: Métodos qualitativo, quantitativo e misto. Penso Editora, 2021.

DA ROCHA RESTELATTO, Marcia; HOFFELDER, Greici Kelly. Comunicação entre familiares e equipe de enfermagem em UTI associada à qualidade da assistência: relato de caso. Anais da Semana Acadêmica e Mostra Científica de Enfermagem, p. 11-11, 2018.

DE SOUZA, Thaise Maia; DE SOUSA LOPES, Graciana. Assistência de enfermagem em terapia intensiva ao paciente com Covid 19: um relato de experiência. Revista Eletrônica Acervo Enfermagem, v. 9, p. e6118-e6118, 2021.

EMANUEL, Ezekiel J. et al. Fair allocation of scarce medical resources in the time of Covid-19. 2020.

FERMINO, Juliana Martins et al. Potencialidades e dificuldades nas práticas de acolhimento na rede de atenção básica conforme a Política Nacional de

Humanização. Saúde \& Transformação Social/Health \& Social Change, v. 6, n. 2, p. 054-069, 2015 
OTHERO, M. B.; DE CARLO, M. M. R. P. A família diante do adoecimento e da hospitalização infantil: desafios para a terapia ocupacional. Prat hosp [Internet]. 2006 [cited 2012 June 06].

HARUNA J, Tatsumi H, Kazuma S, Kuroda H, Goto Y, Aisaka W, Terada H, Masuda Y. Using an ICU Diary to Communicate With Family Members of COVID-19 Patients in ICU: A Case Report. J Patient Exp. 2021 Jul 28;8:23743735211034094. doi: 10.1177/23743735211034094. PMID: 34377772; PMCID: PMC8323441.

LEMMERS, Anne-Lotte; VAN DER VOORT, Peter HJ. Trust in Intensive Care Patients, Family, and Healthcare Professionals: The Development of a Conceptual Framework Followed by a Case Study. In: Healthcare. Multidisciplinary Digital Publishing Institute, 2021. p. 208.

LIPWORTH AD, Collins EJ, Keitz SA, Hesketh PJ, Resnic FS, Wozniak JM, Mosenthal AC. Development of a Novel Communication Liaison Program to Support COVID-19 Patients and Their Families. J Pain Symptom Manage. 2021 Jan;61(1):e1-e10. doi:

10.1016/j.jpainsymman.2020.11.016. Epub 2020 Nov 24. PMID: 33246074; PMCID: PMC7685060.

LIY Y, Cheng Q, Wang JH, Li S, Tian C, Li YX, Zhang WH, Shen N, Qiao J. Family companion between patients with coronavirus disease 2019: a retrospective observational study. Chin Med J (Engl). 2020 Oct 20;133(20):2507-2509.

MOHER, David et al. Reimpressão - itens de relatório preferidos para revisões sistemáticas e meta-análises: a declaração PRISMA. Fisioterapia , v. 89, n. 9, pág. 873- 880, 2009.

MONTUAK TR, Kuhl EA. COVID-related family separation and trauma in the intensive care unit. Psychol Trauma. 2020 Aug;12(S1):S96-S97. doi: 10.1037/tra0000839. Epub 2020 Jun 22. PMID: 32567872.

NUNES, Maurício Rouvel. A atuação do enfermeiro em unidade de terapia intensiva na pandemia de COVID-19: relato de experiência. Revista Eletrônica Acervo Saúde, v. 12, n. 11, p. e4935-e4935, 2020.

OPAS, Organização pan-americana de saúde. Folha informativa sobre COVID-19, 2021. Disponível em: https://www.paho.org/pt/covid19. Acessado 12/05/2021..

PEDROSA, Nathália Lima; ALBUQUERQUE, Nila Larisse Silva de. Spatial analysis of COVID-19 cases and intensive care beds in the State of Ceará, Brazil. Ciência \& Saúde Coletiva, v. 25, p. 2461-2468, 2020.

PISCITELLO GM, Fukushima CM, Saulitis AK, Tian KT, Hwang J, Gupta S, Sheldon M. Family Meetings in the Intensive Care Unit During the Coronavirus Disease 2019 Pandemic. Am J Hosp Palliat Care. 2021 Mar;38(3):305-312.

PROENÇA, Michele de Oliveira; AGNOLO, Cátia Millene Dell. Internação em unidade de terapia intensiva: percepção de pacientes. Revista Gaúcha de Enfermagem, v. 32, n. 2, p. 279-286, 2011.

RAURELL,M. et al; Enfermería Intensiva (English ed.); vol 31; pág. 90-93. Maio-unho; 2020. 
ROTHAN, Hussin A.; BYRAREDDY, Siddappa N. The epidemiology and pathogenesis of coronavirus disease (COVID-19) outbreak. Journal of autoimmunity, v. 109, p.

102433, 2020.

SHARIATI E, Dadgari A, Talebi SS, Mahmoodi Shan GR, Ebrahimi H. The Effect of the Web-Based Communication between a Nurse and a Family Member on the Perceived Stress of the Family Member of Patients with Suspected or Confirmed COVID-19: A Parallel Randomized Clinical Trial. Clin Nurs Res. 2021 Sep;30(7):1098-1106. doi: 10.1177/10547738211017688. Epub 2021 May 28. PMID: 34044625.

Tricco AC, Lillie E, Zarin W, O'Brien KK, Colquhoun H, Levac D, et al. PRISMA Extension for Scoping Reviews (PRISMA-ScR): Checklist and Explanation. Ann Intern Med [Internet]. 2018 Oct 2;169(7):467. Available from: http://www.prisma- statement.org/Extensions/ScopingReviews

VEENENDAAL V N, van der Meulen IC, Onrust M, Paans W, Dieperink W, van der Voort PHJ. SixMonth Outcomes in COVID-19 ICU Patients and Their Family Members: A Prospective Cohort Study. Healthcare (Basel). 2021 Jul 8;9(7):865. doi: 10.3390/healthcare9070865. PMID: 34356243; PMCID: PMC8305246.

WENDEL PK, Stack RJ, Chisholm MF, Kelly MJ, Elogoodin B, Liguori GA, Green DST, Kalsi MS, Soffin EM. Development of a Communications Program to Support Care of Critically III Coronavirus Disease 2019 (COVID-19) Patients. J Patient Exp. 2020 Oct;7(5):673-676. 
